\title{
Von der Euro-Krise zu neuen Ufern? Perspektiven des europäischen Integrationsprojekts
}

Die europäische Integration wird häufig neben dem Sozialstaat als die wichtigste politische Erfindung des 20. Jahrhunderts bezeichnet. Mit ihrer Hilfe ist es in Europa gelungen, die (selbst)zerstörerischen Kräfte des Nationalismus zu bändigen. Der einstige Kontinent der Kriege konnte dadurch in eine dauerhafte Friedens- und Wohlstandszone verwandelt werden. Der Erfolg des Integrationsprojekts war und ist so umfassend, dass andere Weltregionen wie Südostasien und Lateinamerika ihm seit geraumer Zeit nachzueifern versuchen. Allein den Europäern selber scheint das wenig bewusst zu sein, was die Schwierigkeiten belegt, so etwas wie eine gemeinsame europäische Identität auszubilden. Angesichts der immensen Probleme, vor die sich die EU durch die aktuelle Schulden- und Währungskrise gestellt sieht, ist das einerseits verständlich. Auf der anderen Seite verwundert es dennoch, wenn man bedenkt, welche epochalen Fortschritte der Integrationsprozess gerade in den letzten beiden Jahrzehnten gemacht hat. Nicht nur, dass die Friedenszone auf die östliche Hälfte des bis 1989 durch den Ost-West-Konflikt gespaltenen Kontinent ausgeweitet wurde. Auch in der wirtschaftlichen Integration erreichte man durch die Einführung der gemeinsamen Währung eine neue Stufe des Souveränitätsverzichts, die in der im Maastrichter Vertrag vorgenommenen Umbenennung der vormaligen Europäischen Gemeinschaft zur Europäischen Union symbolisch Ausdruck fand.

Nimmt man die unter dem Begriff, „Euroskeptizismus“ zusammengefassten Vorbehalte als Gradmesser, die dem Integrationsprozess heute mehr oder minder explizit entgegengebracht werden, ist die Europäische Union zum Opfer ihrer eigenen Erfolge geworden. Hier liegt übrigens eine interessante Analogie zur anderen großen Innovation des 20. Jahrhunderts - dem Sozialstaat. So wie dieser durch zunehmende finanzielle Überbeanspruchung unter Druck geraten ist, so lässt sich auch die Krise der Europäischen Union als Folge einer Überdehnung beschreiben, die von der gleichzeitigen Erweiterung und Vertiefung der Gemeinschaft in den neunziger Jahren ausging. Diese Krise hat sich mittlerweile so zugespitzt, dass sie für die Substanz des Integrationsprojekts selbst bedrohlich werden könnte.

Nun sind Krisenerfahrungen und -diagnosen für die EU beileibe nichts Neues. Deren ganze Entwicklung kann im Grunde als Abfolge von Krisen gedeutet werden. Tatsächlich wohnt der Krise ja immer auch die Chance zu ihrer Überwindung inne, 
birgt sie die Kraft der Neuerung. In der fünfzigjährigen Integrationsgeschichte lassen sich dafür zahlreiche Belege finden (vgl. Brunn 2002). Als das Projekt einer europäischen Verteidigungsgemeinschaft, das den Nukleus einer Politischen Union hätte bilden sollen, von der französischen Nationalversammlung 1954 zu Grabe getragen wurde, kamen die Staatenlenker der damaligen Sechsergemeinschaft nach einer kurzen Denkpause überein, den Schwerpunkt der Integration auf die Wirtschaft zu verlegen. Nachdem die Zollschranken beseitigt, ein gemeinsamer Agrarmarkt geschaffen und mit dem EWS ein System fester, aber doch anpassungsfähiger Wechselkurse eingerichtet worden war, trat ab Mitte der siebziger Jahre eine erneute Phase der Stagnation ein, die durch die wachsenden Interessengegensätze innerhalb der Gemeinschaft im Zuge der ersten Erweiterungsrunde (1973) zusätzlich befördert wurde. Erst das von Kommissionspräsident Delors angestoßene Binnenmarktprojekt, das bis heute den Kern der marktschaffenden Gemeinschaftspolitiken umschreibt, und die in der Einheitlichen Europäischen Akte von 1986 vereinbarten institutionellen Reformen, die unter anderem die (Wieder-)Einführung der 1966 ausgesetzten Mehrheitsentscheidungen vorsahen, verliehen dem Integrationsprozess wieder Flügel. Sie mündeten in den Maastrichter Vertrag von 1992, der die Einführung der gemeinschaftlichen Währung besiegelte und die EU durch Einbeziehung der bis dahin freihändig koordinierten Außen- und Sicherheitspolitik in das Vertragssystem auch institutionell auf eine neue Grundlage stellte.

Das Binnenmarktprojekt traf damals auf - gemessen an den heutigen Herausforderungen der europäischen Politik - vergleichsweise günstige Bedingungen. Maßgeblich für seinen Erfolg war zum einen, dass von der Beseitigung bestehender Handelsbeschränkungen alle Beteiligten profitierten. Zum anderen bewegte sich die Herstellung der Marktfreiheit in der vorhandenen ,negativen“ Logik des Integrationsprozesses, die den Mitgliedsstaaten zunächst keine großen Kompetenztransfers und spektakulären Souveränitätsverzichte abverlangte. Diese Ausgangslage sollte sich spätestens mit dem Maastricht-Vertrag ändern. Von diesem Zeitpunkt an begann sich nicht nur die Schere zwischen den integrationswilligen und -skeptischen Mitgliedsstaaten in der Union immer mehr zu öffnen. Es traten auch die entdemokratisierenden Wirkungen zunehmend ins Bewusstsein, die der Verlust an unilateraler nationaler Steuerungsfähigkeit im Zuge eines immer rigideren, sich dabei aber ausschließlich auf ,negative“ Integration beschränkenden Binnenmarktregimes mit sich brachte. 


\section{Erweiterung und Vertiefung: ein Widerspruch?}

In der öffentlichen Kommentierung wird die Überdehnung der Europäischen Union weniger mit ihrer Vertiefung als mit ihrer Erweiterung in Verbindung gebracht. Tatsächlich hat der Beitritt von 12 Ländern Mittelosteuropas - die vierte Erweiterungsrunde der einstigen Sechser-Gemeinschaft - alle früheren Beitritte in den Schatten gestellt. Das Interessengefälle innerhalb der Union ist damit gewachsen. Nicht nur, dass zwischen den alten und neuen Mitgliedsstaaten immense Unterschiede in der Wirtschaftskraft bestehen. Die Integrationswilligkeit der Beitrittsländer wird auch dadurch auf die Probe gestellt, dass diese nach Ende der Sowjetherrschaft wenig Zeit hatten, sich als klassische Nationalstaaten zu konsolidieren, bevor sie mit dem von ihnen selbst herbeigesehnten EU-Beitritt neuerlichen Souveränitätsverzicht leisteten. Daraus den Schluss zu ziehen, eine (weitere) Vertiefung der Union werde durch die MOE-Staaten verhindert oder erschwert, wäre aber verkehrt. Diejenigen, die solche Auffassungen vertreten, gehören in der Regel zu den Befürwortern eines Europas unterschiedlicher Geschwindigkeiten. Mit der Währungsunion, an der 17 der insgesamt 27 EU-Staaten teilnehmen, oder dem SchengenAbkommen ist dieses Konzept ja schon heute Realität. Überdehnungen finden freilich auch hier statt. So wie Griechenland - bei ernsthafter Beachtung der ökonomischen Kriterien - nicht in die Euro-Zone hätte aufgenommen werden dürfen, so stellen Schengen-Mitglieder wie Dänemark oder Italien die Abschaffung der Binnengrenzen heute wieder in Frage. Dabei handelt es sich nicht zufällig um Länder, deren Regierungen unter starkem Druck rechtspopulistischer Kräfte stehen. Umgekehrt kann aus dem Vorhandensein und der Relevanz solcher Kräfte in den Beitrittsländern nicht gefolgert werden, dass deren Bevölkerungen und Eliten der europäischen Integration ebenso skeptisch (oder noch skeptischer) gegenüberstünden. In manchen Politikfeldern - etwa der Außenpolitik (siehe unten) - ist eher das Gegenteil der Fall (Maćków 2009). Und auch im Zusammenhang mit der Schuldenkrise war es mehr als verständlich, dass Länder wie die Slowakei sich mit der gemeinschaftlichen Solidarität schwer taten, hatte man ihren Bevölkerungen im Zuge des Transformationsprozesses doch große Opfer zugemutet. Warum sollten sie jetzt für die Schulden der Länder geradestehen, die solche Opfer nicht erbringen mussten?

Berechtigt ist die Rede von der Erweiterungskrise, wenn man sie auf die institutionelle Struktur der EU bezieht. Hier liegt die Verantwortung aber ebenfalls weniger bei den neuen als bei den alten Mitgliedsstaaten, deren Regierungen unfähig waren, die notwendigen Anpassungen vor dem Beitritt vorzunehmen. Wurde bei den früheren Runden ausschließlich nach der Aufnahmefähigkeit der Beitrittsländer 
gefragt, so ging es bei der Osterweiterung erstmals auch um die Aufnahmefähigkeit der EU selbst. Dass eine bloße Fortschreibung der alten institutionellen Strukturen für die Erweiterung nicht genügen würde, hätte den Staats- und Regierungschefs eigentlich klar sein müssen, doch fehlte ihnen die Kraft und der Wille, gemäß dieser Einsicht zu handeln. Die Konsequenz war der dilettantisch ausgehandelte und inhaltlich völlig unzureichende Vertrag von Nizza, der für den nachfolgenden Verfassungsprozess eine schwere Bürde darstellte. Man muss keine besonders hohen Maßstäbe anlegen, um die Antworten, die der Lissabon-Vertrag für die institutionellen Probleme der EU der 27 gefunden hat, „suboptimal“ zu finden. Aus ihnen erwächst weder eine Stärkung der Handlungsfähigkeit der Gemeinschaft, noch können sie deren demokratisches Defizit lindern. Zusätzlich delegitimiert wurde das Vertragswerk durch die kaum verhohlene Absicht der Staats- und Regierungschefs, die neu geschaffenen Ämter eines permanenten Ratspräsidenten und eines dem Rat und der Kommission gleichzeitig zugeordneten Außenbeauftragten bewusst mit schwachen Persönlichkeiten (dem Belgier Herman Van Rompuy und der Britin Catherine Ashton) zu besetzen, die ihre eigenen Kreise nicht stören würden.

Das Unvermögen der europäischen Staatenlenker verweist auf die Krise der sogenannten intergouvernementalen Methode. Herrschte bis zum Maastricht-Vertrag ein annäherndes Gleichgewicht zwischen den Gemeinschaftsorganen Kommission und Parlament auf der einen und den nationalen Regierungen auf der anderen Seite, so sind mit der Etablierung einer zweiten und dritten Integrationssäule in der Außenund Sicherheits- sowie Innen- und Justizpolitik die Regierungen als europäische Entscheidungsträger immer mehr in den Vordergrund getreten. Die nachfolgenden Vertragsänderungen (von Amsterdam über Nizza bis Lissabon) haben diese Struktur perpetuiert. Der Vorrang der intergouvernementalen Methode läuft darauf hinaus, dass der Konsens der Mitgliedsstaaten die wichtigste Voraussetzung für nennenswerte Integrationsfortschritte bleibt. Einen solchen Konsens herbeizuführen war schon in der Union mit 15 Mitgliedern schwierig. In einer Union mit 27 oder 30 Mitgliedern kann es nur funktionieren, wenn das Einstimmigkeitsprinzip durch qualifizierte Mehrheitsentscheidungen weitgehend ersetzt wird. In Schlüsselbereichen der nationalen Souveränität ist das bis heute nicht der Fall. Das Vetorecht bleibt nicht nur im gesamten Feld der Außenpolitik bestehen. Es greift auch bei souveränitätsrelevanten Fragen der Wirtschaftspolitik wie den auf dem Brüsseler Gipfel im Juli 2011 geschaffenen Möglichkeiten einer Kreditvergabe durch den Europäischen Rettungsschirm (ESM), mit dem die EU faktisch den Einstieg in eine Transfer- und Haftungsunion vollzogen hat.

Das Festhalten am Vetorecht und die Ausdehnung der wirtschaftspolitischen Befugnisse der Union sind Seiten derselben Medaille. Sie halten den Anschein natio- 
naler Souveränität aufrecht, verschärften aber in Wahrheit das demokratische Defizit. Die nationalen Parlamente können die haushaltsrelevanten Beschlüsse der europäischen Institutionen längst nicht mehr wirksam kontrollieren geschweige denn vorsorglich beeinflussen, mag das deutsche Verfassungsgericht das Gegenteil auch noch so oft postulieren. Die ehrliche Konsequenz daraus müsste im Grunde lauten, dass man die haushaltsrelevanten Beschlüsse denselben Entscheidungsverfahren unterwirft wie die Gemeinschaftspolitiken des Binnenmarktes. Die Kontrolle würde dann auf der Ebene stattfinden, auf der die Entscheidungen tatsächlich getroffen werden.

Kritiker haben zu Recht darauf hingewiesen, dass eine Währungsunion auf Sand gebaut ist, wenn man weiterhin national unterschiedliche Wirtschaftspolitiken betreibt. Dies gilt ganz unmittelbar für die Fiskalpolitik, trifft aber auch auf die Lohnund Wachstumspolitik zu, von denen die Wettbewerbsfähigkeit eines Landes maßgeblich abhängt. Die sogenannte No-Bailout-Klausel des Maastrichter Vertrags, nach der Staaten nicht für die Schulden anderer Staaten haften, ist durch die weltweite Finanzkrise als wirklichkeitsfremd entlarvt worden. Wenn Länder wie Griechenland bei der Finanzierung ihrer Schulden ganz auf sich allein gestellt bleiben, indem sie deren Bedienung nur über die Finanzstärke sicherstellen können, werden die Zinsen für die fälligen Anleihen bei Zweifeln an der Zahlungsfähigkeit automatisch in die Höhe getrieben. Nach den Brüsseler Beschlüssen kann der europäische Krisenfonds solche Anleihen künftig aufkaufen bzw. umtauschen, was für die angeschlagenen Länder eine Minderung ihrer Schuldenlast bedeutet.

Die Kehrseite der „Haftungsunion“ besteht darin, dass sie die Anreize der potenziellen Nehmerländer noch weiter vermindert, eine solide Haushaltspolitik zu betreiben. Für die Stabilität der Währungsunion wird daher entscheidend sein, ob sie der notwendigen Haushaltsdisziplin durch ein besseres Schuldenregime den Weg bahnt. Der Geburtsfehler des Euro lag ja nicht darin, dass es an entsprechenden Regeln gefehlt hätte - es fehlte allein an der Bereitschaft, auch Deutschlands und Frankreichs, sich an diese Regeln zu halten. Griechenland zahlt für seinen Verbleib in der Währungsunion jetzt einen hohen Preis: Seine Haushaltspolitik wird unter gemeinschaftliche Kuratel gestellt, was einer weitgehenden Aufgabe der staatlichen Souveränität gleichkommt. Zwar ist es schwer vorstellbar, dass andere Staaten ein ähnliches Schicksal in Kauf nehmen würden. Dennoch bleiben Zweifel, ob die 
Durchsetzung strikter Schuldenregeln in Europa unter den Bedingungen der Einstimmigkeit erreicht werden kann. ${ }^{1}$

\section{Von der negativen zur positiven Integration}

Ein grundsätzliches Legitimationsproblem Europas rührt aus der wirtschaftlichen manche meinen sogar „,neoliberalen“ - Schlagseite des Integrationsprozesses. Sie ist der Hauptgrund, warum ein gemeinsames Identitätsbewusstsein in der EU bislang nicht herangereift ist. Die eigentlichen Gemeinschaftspolitiken konzentrieren sich bis heute auf die Schaffung und Regulierung des gemeinsamen Marktes; die Bewältigung der kulturellen, sozialen und ökologischen Nebenfolgen, die sich aus dem Marktgeschehen ergeben, bleibt ebenso ausgespart (und wird den Mitgliedsstaaten überlassen) wie die Außen-, Sicherheits- und Verteidigungspolitik. Auch die wirtschaftliche Integration ist - wie gesehen - auf eigentümliche Weise halbiert, indem der vollständig vergemeinschafteten Geldpolitik das Fehlen von Zuständigkeiten in der Fiskal-, Wachstums-, Konjunktur- und Arbeitsmarktpolitik gegenübersteht. Diese Politikbereiche werden durch Zielvereinbarungen - etwa im Rahmen der sogenannten Lissabon-Strategie - unter den Mitgliedsstaaten bestenfalls lose koordiniert. Selbst in Bereichen wie der Energiepolitik, deren globale strategische Implikationen die EU eigentlich auf ein gemeinsames Handeln verpflichten müssten, denken und handeln die Regierungen weiterhin fast ausschließlich in nationalstaatlichen Kategorien.

In Abwandlung eines bekannten Diktums von Daniel Bell könnte man sagen, dass sich die EU um die kleinen Probleme zuviel und um die großen zuwenig kümmert. Natürlich wird es immer unterschiedliche normative Vorstellungen geben, wie eine sinnvolle Kompetenzaufteilung zwischen der nationalen und der supranationalen Ebene aussehen sollte. Doch würden auch (oder gerade) die Kritiker einer weiteren Vertiefung der Integration, die beispielsweise den Einstieg in eine Transferunion ablehnen, es als Schlüsselfrage der Zukunft Europas betrachten, ob die Gemeinschaft der 27 oder mehr Staaten sich in der globalisierten Welt als kollektiver Akteur behauptet. Die zentrale Herausforderung besteht mit anderen Worten in der Entwicklung einer gemeinsamen Außen- und Sicherheitspolitik, die diesen Namen tatsächlich verdient. Gerade sie könnte identitätsstiftend wirken, da sie die Einheit des Staates nach innen und außen am ehesten verkörpert. In bundesstaatlichen Ordnungen wird die Außen- und Verteidigungspolitik deshalb traditionell von der Zentral-

1 Deutschland wäre inzwischen bereit, einen durch die Kommission in Gang zu setzenden automatischen Sanktionsmechanismus zu akzeptieren, der nur von einer qualifizierten Mehrheit ausgehebelt werden könnte. Diese Lösung scheiterte bis zuletzt am Widerstand Frankreichs. 
gewalt wahrgenommen. Der Staatenverbund der EU hat dieses Prinzip im bisherigen Verlauf des Einigungsprozesses auf den Kopf gestellt.

Die Schlagseiten der Integration liefern auch einen Zugang zum häufig beklagten Demokratiedefizit der EU. Umfragen zeigen, dass sich eine Mehrheit der Bevölkerung in den Mitgliedsstaaten eine gemeinsame Außenpolitik wünscht; die Integration scheitert hier offenbar weniger am Volk als an den nationalen Eliten, die diese Spielwiese nicht ohne Not preisgeben möchten. Etwas anders verhält es sich auf den verschiedenen Feldern der Sozial- und Wirtschaftspolitik. Im Unterschied zur Außenpolitik, die die Lebenswirklichkeit der Menschen zumeist nicht direkt berührt, handelt es sich hier um Fragen von hoher elektoraler und legitimatorischer Relevanz. Entscheidungen über Sozialleistungen, Steuern, Subventionen usw. erzeugen in der Regel Verteilungswirkungen und sind deshalb besonders konfliktträchtig. In der Europaliteratur wird dies häufig als Grund dafür genannt, warum man die Verantwortung für diese Bereiche nicht von der nationalen auf die europäische Ebene übertragen könne.

Auch das ist aber nur die halbe Wahrheit. Wenn es stimmt, dass sich demokratische Legitimität sowohl aus den Möglichkeiten der Teilnahme am politischen Prozess speist als auch aus den Ergebnissen, die dieser Prozess hervorbringt, könnte das Fehlen EU-weiter Standards in der Sozial- und Wirtschaftspolitik von der Bevölkerung durchaus als Problem empfunden werden. Ein Beispiel ist das vergebliche Bemühen der Europäischen Kommission, die Finanzierung des im Vergleich zu den nationalen Budgets nach wie vor bescheidenen EU-Haushalts durch Einführung einer Gemeinschaftssteuer (etwa auf Finanztransaktionen) auf eine von den Mitgliedsstaaten unabhängigere Basis zu stellen. Eine solche Steuer wäre nicht nur ökonomisch sinnvoll, sie würde nebenbei auch das europäische Gemeinschaftsgefühl verstärken und damit ähnlich wirken wie die Währungsunion oder eine gemeinsame Außen- und Sicherheitspolitik. Dass sie bisher nicht zustandegekommen ist, liegt wohl kaum an den Bürgern. Dies verweist auf ein Kernproblem der europäischen Mehrebenendemokratie. Weil demokratische Strukturen auf der EU-Ebene noch nicht hinreichend ausgebildet sind oder funktionieren, müssen die elektoralen Konsequenzen der europäischen Politik nahezu ausschließlich von den Mitgliedsstaaten getragen werden. Den politischen Akteuren kommt das durchaus gelegen, weil sie dadurch die Möglichkeit gewinnen, Europa für die Versäumnisse und Fehler der eigenen Politik die Schuld zuzuweisen. Das institutionelle Demokratiedefizit der EU leistet dabei willkommene Dienste, hilft es ihnen doch, ihre Beteiligung am Zustandekommen (oder Nicht-Zustandekommen) der europäischen Entscheidungen zu verschleiern. So tragen die nationalen Politiker selbst dazu bei, die europä- 
ische Politik zu delegitimieren, nur um die negativen Konsequenzen dieser Delegitimierung anschließend lauthals zu beklagen (Decker 2000).

Die empirischen Belege der Missstimmung müssen hier nicht im Einzelnen aufgelistet werden: notorisch niedrige und weiter sinkende Beteiligungen bei den Wahlen zum Europäischen Parlament, wachsender Zuspruch anti-europäischer Parteien, rückläufige Zustimmungswerte zur eigenen EU-Mitgliedschaft seien an dieser Stelle nur exemplarisch genannt. Der negative Ausgang der Referenden zum Verfassungs- bzw. Lissabon-Vertrag in Frankreich, den Niederlanden und Irland hat die Krise nur manifestiert. Hätte man Volksabstimmungen in weiteren Ländern zugelassen, wäre die Zahl der Neinsager sicher noch größer gewesen. Besonders nachdenklich muss stimmen, dass die EU auch in Ländern Verdrossenheit erzeugt, die von der EU-Mitgliedschaft massiv profitiert haben - etwa in Österreich, wo über der Frage, wie man mit dieser Anti-Haltung am besten umgeht, 2008 sogar die Regierung zerbrochen ist. Angesichts dieser Stimmungslage ist es gut nachvollziehbar, dass unter den Staats- und Regierungschefs zur Zeit niemand den Mut entwickelt, das Integrationsprojekt offensiv voranzubringen. Stattdessen triumphierte auf dem Brüsseler Gipfel einmal mehr das Prinzip des „sich Durchwurschtelns“, das die EU in ihre heutige Legitimationskrise mit hineingeführt hat.

\section{Welches Europa wollen wir?}

Welche Konsequenzen sind daraus zu ziehen? Die Debatte, die vor dem Hintergrund der Schuldenkrise in den vergangenen Monaten in den bundesdeutschen Feuilletons geführt worden ist, hat nochmals offengelegt, wie sehr die normativen Grundpositionen zu Europa und zur Europäischen Union inzwischen auseinanderklaffen. Die Trennlinie verläuft dabei nicht nur zwischen den Befürwortern und Skeptikern einer weiteren Integration. Auch innerhalb der beiden Lager lassen sich jeweils zwei Gruppen bzw. Richtungen voneinander unterscheiden: eine basisdemokratische oder sogar offen populistische und eine elitenbezogene.

Eine intellektuelle Begründung des euroskeptischen Populismus versucht der Schriftsteller Hans Magnus Enzensberger zu liefern (2011). Sein altbekanntes Lamento vom bürokratischen Brüsseler Moloch, das die EU mit Vorschriften über die Länge und Krümmung von Gurken gleichsetzt, orientiert sich an Maßstäben der demokratischen Input-Legitimation, vor denen die EU naturgemäß nicht bestehen kann. Zudem wird übersehen, dass das demokratische Defizit der EU - wie gesehen - hauptsächlich von den in ihrem nationalen Horizont befangenen Regierungen der Mitgliedsstaaten ausgeht und nicht von den supranationalen Institutionen. An beiden Punkten widerspricht Peter Graf Kielmansegg, der innerhalb der euroskepti- 
schen Position den „realistischen“ Gegenpol zu Enzensberger vertritt. Unter Rückgriff auf sein Theorem der „Erinnerungs-, Erfahrungs- und Kommunikationsgemeinschaft" (1996: 55) bezweifelt Kielmansegg, dass Europa die Voraussetzungen für einen Integrationssprung auf dem Feld der Währungs- und Wirtschaftspolitik mit sich bringe. Er warnt die Staatenlenker deshalb davor, den Bürgern einen solchen Sprung zu oktroyieren - nach dem Motto, dass ,wenn man sich tief genug in die Krise hat hineinziehen lassen, (es) keinen anderen Ausweg aus der Krise mehr zu geben scheint.“ (2011: 35)

Unter den Befürwortern einer weiteren Vertiefung der Integration steht Jürgen Habermas am unbeirrtesten für die demokratieoptimistische Spielart. Habermas geht gegen die These des institutionellen Demokratiedefizits davon aus, dass die EU über den Verfassungsrahmen längst verfüge, um die zwischen den Mitgliedsstaaten und der Gemeinschaft geteilten Kompetenzen nicht nur zu verrechtlichen, sondern demokratisch zu verrechtlichen. Bedingung dafür sei freilich eine Europäisierung der nationalen Öffentlichkeiten, die von den politischen Eliten befördert werden müsste. „Stattdessen beobachten wir auf Seiten der Regierungen - dafür ist die deutsche Kanzlerin zur Symbolfigur geworden - ein hinhaltendes Taktieren und auf Seiten der Bevölkerungen eine populistisch geschürte Ablehnung des europäischen Projekts im ganzen.“ (Habermas 2011: 48) Herfried Münkler (2011) teilt Habermas' Kritik an den mutlosen Eliten, nicht aber dessen Zutrauen in das demokratiefördernde Potenzial einer aufgeklärten Zivilgesellschaft. Den Versuch, das Elitenversagen in der EU durch eine konsequente Demokratisierung zu bekämpfen, hält er sogar für ausgesprochen fahrlässig. Fortschritte seien nur von anderen und besseren Eliten zu erwarten, die allerdings erst hervorgebracht werden müssten. Als Voraussetzung dafür betrachtet Münkler die Schaffung eines kleineren, handlungsfähigeren Zentrums europäischer Kernstaaten, womit sich die Analyse gewissermaßen im Kreis dreht. Denn auch dieses Kerneuropa müsste ja erst (und von wem?) auf den Weg gebracht werden.

\section{Literatur}

Brunn, Gerhard, 2002: Die Europäische Einigung von 1945 bis heute, Stuttgart.

Decker, Frank, 2000: Demokratie und Demokratisierung jenseits des Nationalstaates. Das Beispiel der Europäischen Union, in: Zeitschrift für Politikwissenschaft 10 (2), 585-629.

Enzenzberger, Hans Magnus, 2011: Sanftes Monster Brüssel oder Die Entmündigung Europas, Frankfurt a. M. 
Habermas, Jürgen, 2011: Wie demokratisch ist die EU? Die Krise der Europäischen Union im Licht einer Konstitutionalisierung des Völkerrechts, in: Blätter für deutsche und internationale Politik 66 (8), 37-48.

Kielmansegg, Peter Graf, 1996: Integration und Demokratie, in: Markus Jachtenfuchs/Beate Kohler-Koch (Hrsg.), Europäische Integration, Opladen, 47-71.

Kielmansegg, Peter Graf, 2011: Soll von Demokratie noch die Rede sein?, FAZ, 8.7.2011, 35 .

Macków, Jerzy, 2009: Europäismus. Warum die Europäische Union demokratisiert werden muss und eine gemeinschaftliche Außenpolitik braucht, in: Frank Decker/Markus Höreth (Hrsg.), Die Verfassung Europas, Wiesbaden, 295-318.

Münkler, Herfried, 2011: Alle Macht dem Zentrum. Warum eine Demokratisierung Europa nicht retten wird, Der Spiegel, 4.7.2011 (27), 108 f.

Korrespondenzanschrift:

Prof. Dr. Frank Decker

Institut für Politische Wissenschaft und Soziologie

Rheinische Friedrich-Wilhelms-Universität Bonn

Lennéstraße 27

53113 Bonn

E-Mail: frank.decker@uni-bonn.de 